\title{
Garten - Kabinett - Schlachtfeld. Räume des empfindsamen Briefes
}

\section{Zur Einführung}

Im Juli 1772 brechen der Naturforscher Johann Reinhold Forster und sein Sohn Georg mit Kapitän James Cook zur zweiten Weltumsegelung auf. Erste große Station macht man im November desselben Jahres am Kap der Guten Hoffnung. Obgleich der Schiffsalltag auf der Resolution und die naturkundlichen Beobachtungen an Land vornehmlich in diaristischen Aufzeichnungen Johann Reinhold Forsters sowie in den Zeichnungen von Georg Forster dokumentiert sind, findet man Zeit, zahlreiche Briefe an Gelehrte in ganz Europa zu verfassen. ${ }^{1}$ Für Naturforschende und globale Akteure wie die Forsters ist der Brief Teil ihrer Existenz, auch und gerade weil er als äußerst mobile Form an die Schreiborte bzw. Räume des reisenden Forschers angepasst werden kann. Informationen über neu entdeckte und beobachtete Räume, deren Pflanzen und Tiere, die Forster gemeinsam mit seinem Sohn auf den Zwischenstationen in Madeira und während ihres Aufenthaltes am Kap sammelt, werden per Brief als eine Art verschriftlichter Kollektion an Gelehrte wie Carl von Linné übersendet; wobei der Brief nicht nur als Träger von Text, sondern auch als Übermittler von nichtsprachlichen Informationen - etwa die zahlreich beigegebenen und mitgesendeten naturkundlichen Objekte - funktioniert. Am Anfang eines Briefes an Linné lässt sich der als spröde geltende Forster zu den poetischen, die Landschaft geradezu segnenden Worten hinreißen: „Felix prae aliis regio, affluens Florae, Faunaeque, divitiis magnam portes in explorari.“2 Zugleich avanciert der Brief zum exklusiven Textträger von Erkenntnis und zu einem eigenständigen ,taxonomischen Raum'. Mit Bezug auf eine Schrift Walter Benjamins über die „tiefste Bezauberung“3 des Sammelns ließe sich formulieren, dass der Brief zu einer Art virtuellen Vitrine wird, durch die der Sammler, der hier

1 Vgl. dazu Kittelmann 2020a.

2 Übers.: „Vom Glück (vor allen anderen) begünstigte Landschaft, gesegnet mit Flora und Fauna, mögest Du den Reichtümern den großen [Reichtum] des Unerforschten hinzufügen.“

3 Benjamin 1991, 389.

Jana Kittelmann, Martin-Luther-Universität Halle-Wittenberg

Ә Open Access. (c) 2021 Jana Kittelmann, publiziert von De Gruyter (cc) BY-NC-ND Dieses Werk ist lizenziert unter der Creative Commons Attribution-Non-Commercial-NoDerivatives 4.0 International Lizenz. https://doi.org/10.1515/9783110712568-007 


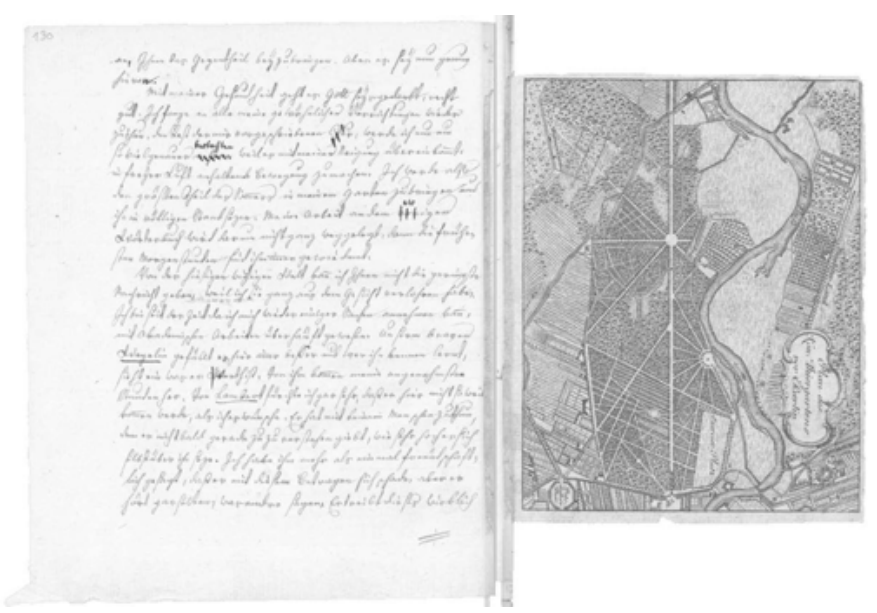

Abb. 1: In einem Schreiben an Johann Jakob Bodmer mitgesandter Plan von Johann Georg Sulzers Berliner Garten, 1764.

nun der Briefempfänger ist, in die Ferne schauen und bislang nie gesehene Räume erkunden und entdecken kann.

Nicht auf einem Schiff, sondern in seinem Halberstädter Garten empfängt hingegen Johann Wilhelm Ludwig Gleim zwei Jahrzehnte zuvor einen Brief seines Freundes Ewald Christian von Kleist: „Aber nun muß ich Ihnen doch sagen, wie mich Ihr liebstes Schreiben antraf. Im Garten unter meinem liebsten Kirschbaum mit einem neueren französischen Poeten, in der Hand und mehr in Gedanken an meinen Kleist als beim Lesen. “4 Empfindsames Natur- und Gartenideal, sentimentaler Freundschaftskult, zeitgenössische Lesestoffe und gesellige Lektürepraktiken fallen ausdrücklich zusammen, wenn Gleim im selben Jahr an Johann Peter Uz schreibt: „Bisher habe ich mich mit Anlegung eines Gartens beschäftiget, und in einer recht königlichen Laube sehr oft geseßen, mit Horaz oder Uz in der Hand." ${ }^{5}$

Dass der Schreibort und die räumliche Umgebung direkten Einfluss auf die Briefwechsel ausüben konnten, zeigt auch ein Brief des Ästhetikers, Philosophen und Naturforschers Johann Georg Sulzer an seinen Freund und Mentor Johann Jakob Bodmer in Zürich. Sulzer sendet Bodmer häufig Briefe aus seinem in Moabit (bei Berlin) gelegenen Garten, schwärmt von den Betätigungen „unter freyem

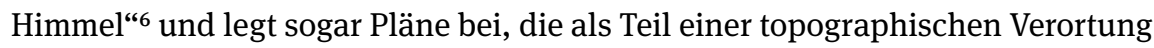

4 Johann Wilhelm Ludwig Gleim an Ewald Christian von Kleist, 27. April 1757, in: Kleist 1881, Bd. 3 , 197.

5 Gleim an Johann Peter Uz, 16. August 1757, in: Gleim u. Uz 1899, 221.

6 Johann Georg Sulzer an Johann Jakob Bodmer, 29. Mai 1775, in: Sulzer u. Bodmer 2020, 967. 
beim Adressaten großen Eindruck machen. So lässt sich der eher am Schreibpult anzutreffende Buchgelehrte Bodmer prompt dazu anregen, in seinen Briefen eine Art zu beackernde Agrarlandschaft zu sehen: „Papier ist mein Feld, Federn die Pflugschar, Dinte oder Witz der Dünger“, schrieb er an Sulzer, ${ }^{7}$ dessen Garten er außerdem in einer Ode verewigt.

Zwischen Garten und Kabinett, Innen und Außen bewegt sich hingegen eine Äußerung Goethes an Johann Caspar Lavater vom 19. Februar 1777. Mit der Anmerkung „Nachts in meinem Garten, in einem warmen Stübgen, da mir draußen über Schnee und hellen Mondenschein, Waldhörner übers Thal herrüber blasen“, 8 changiert Goethe zwischen einer konkreten Ortsangabe und einer die eigene Gemütslage widerspiegelnden Situationsevokation.

Nahezu verstörend trist erscheint dagegen der Raum, in dem sich der junge Theodor Fontane nach seinem Ausscheiden aus dem Diakonissenhaus Bethanien 1849 wiederfindet. Seinem Freund Bernhard von Lepel entwirft Fontane ein düsteres Bild seines „Chambre garni“ mit einer „knarrende[n] Bettstelle, die mitleidsvoll aus den Fugen geht, um einer obdachlosen Wanzenfamilie ein Unterkommen zu bieten.“ Sein Brief schließt mit einem ironisch-verzweifelten „O, es ist schön!“9 Fanny Lewald, erfolgreiche Kollegin Fontanes und eine der ersten Berufsschriftstellerinnen des 19. Jahrhunderts, ist von ihrem Zimmer in London, wo sie sich 1850 für ein Buchprojekt aufhält, dagegen überaus angetan. Ihrem Geliebten (und späteren Ehemann) Adolf Stahr übermittelt sie genauestens Lage, Ausstattung und Interieur und fertigt darüber hinaus eine Skizze an, die sie ihrem Brief beilegt (Abb. 2).

Dass gerade in Briefen geschilderte Räume einem Anspruch der konkreten Erfahr- und Kartierbarkeit folgen, zeigen auch Briefwechsel wie der zwischen Hermann von Pückler-Muskau und seiner Familie. Pückler legt nicht nur Pläne und Zeichnungen der von ihm in der Fremde besuchten Gärten und Landschaften bei, sondern sendet Objekte und Zeichen etwa in Form von gesammelten und gepressten Blüten aus den aufgesuchten Räumen mit, die sowohl als emotionales Zeichen als auch Studien- und Anschauungsobjekt verstanden werden können (Abb. 3).

Zugegeben, es handelt sich hierbei um eine willkürliche Auswahl an Akteurinnen und Akteuren, die verschiedene Phasen der Briefkultur geprägt und in unterschiedlicher Art und Weise an deren Geschichte mitgeschrieben haben. Der Anlass der Briefe, die Bindungen und Beziehungen zwischen den Korrespondenzpartnern

\footnotetext{
7 Bodmer an Sulzer, 16. Mai 1775, in: Sulzer u. Bodmer 2020, 965.

8 Goethe 1997, 81.

9 Fontane u. Lepel 2006, 162.
} 


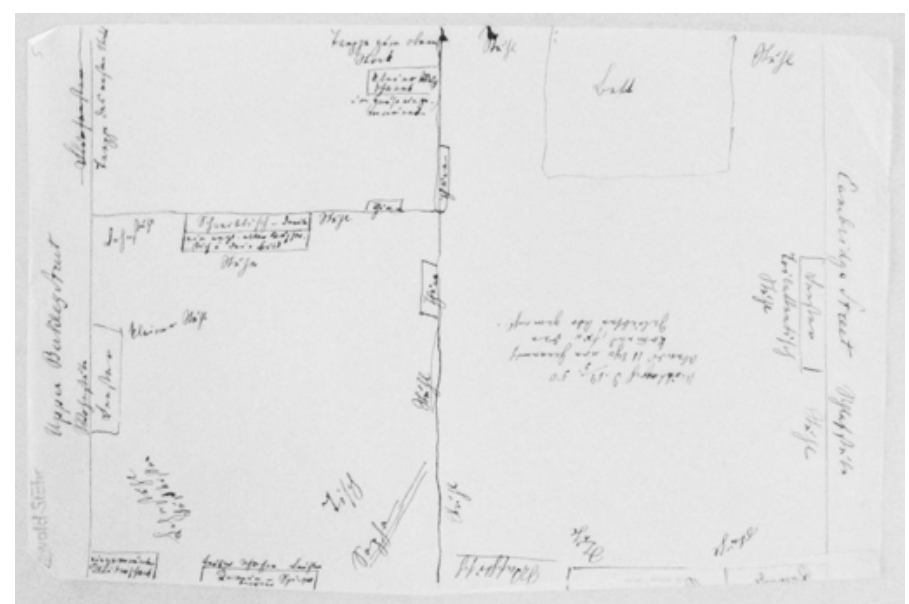

Abb. 2: Skizze ihres Zimmers in einem Brief Fanny Lewalds an Adolf Stahr, 1850.

und -partnerinnen sind unterschiedlich und reichen von gelehrtem Austausch über gesellige Freundschaften bis hin zu intimen Liebesbriefwechseln. Was jedoch auffällt, und darum soll es im Folgenden gehen, ist der verbindende Aspekt des Entwurfes, der Darstellung, der Beschreibung und auch der Inszenierung spezifischer Räume. Ob sie nun als Wissens- und Erkenntnisraum, als je nach Gefühlsage empfindsames oder topographisches Abbild des eigenen Innenlebens oder als emotionaler Liebesraum präsentiert werden - in jedem der hier angeführten Beispiele erscheinen die Schreib- und Lektüreorte als Bedeutungsträger, denen unterschiedliche kulturelle, soziale und ästhetische Konzepte und Modelle von ,Raum' eingeschrieben sind. Das regt zu Überlegungen an, welche Rolle Räume und Topographien für und in Briefen und Briefwechseln spielen.

Dabei geht es in den folgenden Ausführungen nicht um ein mathematisches oder philosophisches Verständnis von Raum. Vielmehr folgt der Beitrag einem kulturgeschichtlichen Ansatz, in dem der (epistolare) Raum als Teil gesellschaftlicher, sozialer und literarischer Interaktion verstanden wird. Das Thema des Bandes aufgreifend, stellt sich die Frage, ob, und wenn ja, wie, die Geschichte des Briefes auch als eine Geschichte der Räume, in denen Briefe verfasst werden, zwischen denen sie vermitteln und hin und hergehen, die sie entwerfen, ritualisieren und auch brechen, erzählt werden kann.

Nun ließe sich freilich einwenden, dass die Frage trivial sei. Schließlich erwartet man gerade von Briefen, dass sie als Lebenszeugnisse in einem lebensweltlichen Raum ihrer Verfasser und Verfasserinnen, Adressaten und Adressatinnen situiert sind, dass sie als mobile und dynamische Textträger zwischen Räumen vermitteln, 


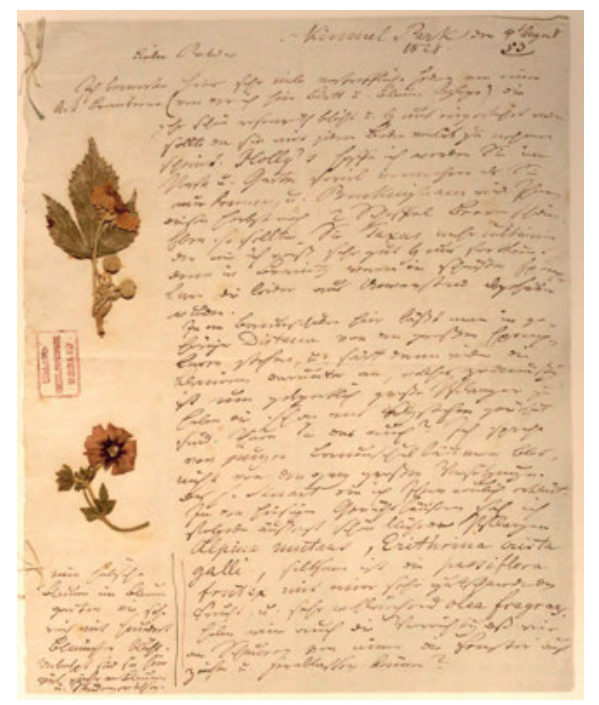

Abb. 3: Brief Hermann von Pückler-Muskaus aus England mit getrockneten Pflanzen an seinen Gärtner Johann Jacob Rehder.

dass ihnen wie keinem anderen Medium die Spuren ihrer Entstehungsorte und topographischen Entstehungssituationen eingeschrieben sind. Das betrifft nicht zuletzt das epistolare Material im Allgemeinen, das häufig durch Datierung, Poststempel, Briefpapier oder Briefbögen selbst Rückschlüsse auf Entstehungsorte gibt und zulässt (Abb. 4).

Dennoch scheint es nicht zuletzt vor dem Hintergrund des spatial turn oder der sogenannten räumlichen oder topographischen Wende, die seit einigen Jahren die Kultur- und auch die Literaturwissenschaften bewegt, nicht uninteressant zu sein, den Raum als epistolare Kategorie ins Blickfeld zu rücken, und zwar insofern, dass Briefe nicht nur auf Welten referieren, sondern diese in unterschiedlicher Weise eben auch modellieren. So ließe sich fragen, in welchen Zeiten und Epochen bestimmte Räume in Briefen Konjunktur haben? Gibt es so etwas wie epochenübergreifende konstante topographische Konstellationen? Wann sind Räume so etabliert, dass sie bzw. das epistolare Agieren in ihnen ritualisiert wird? Wann finden wir zum ersten Mal bestimmte Schreiborte wie das Schiff, das Kabinett oder den Garten und Interieurs wie etwa das Pult oder den Schreibtisch in Briefen und welche Rolle spielen sie fortan für die epistolare Konzeption, Evokation und Imagination? Inwiefern sind architektonische Aspekte und städtebauliche Entwicklungen von Bedeutung? Gibt es Schnittstellen zu anderen Medien und Gattungen? Sind Untersuchungen zum Raum und zur Räumlichkeit in der fiktionalen Literatur 


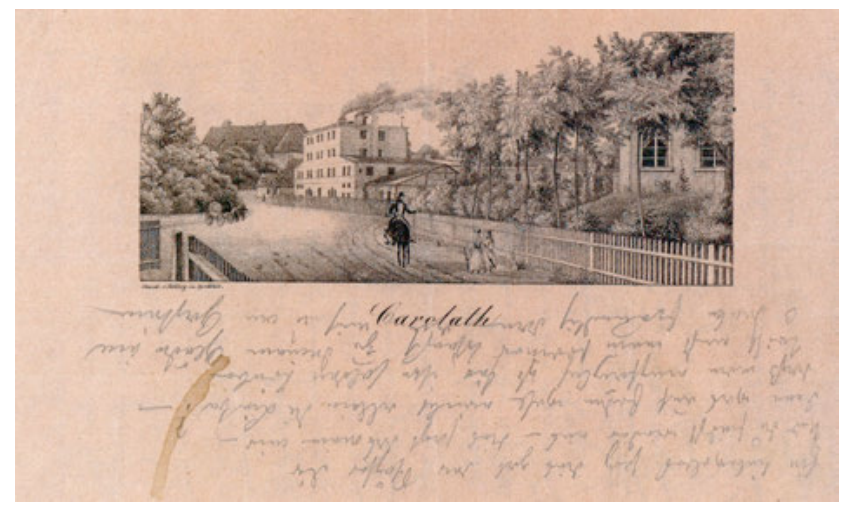

Abb. 4: Brief der Fürstin Adelheid von Carolath auf Carolather Briefpapier.

mittlerweile fest in der Forschung etabliert, ${ }^{10}$ so scheint dagegen für Briefe noch nicht alles gesagt zu sein, obgleich etwa die Ausführungen Jörg Schusters zum epistolaren Interieur um 1900,11 Beiträge von Wolfgang Bunzel zu Schreibszenen in Briefen ${ }^{12}$ oder Ingo Breuers Bemerkungen zu Topographien weiblicher Briefkultur im 18. Jahrhunder $\mathrm{t}^{13}$ bereits grundlegende Denk- und Interpretationsansätze geliefert haben. Um methodisch und systematisch verbindliche Aussagen treffen zu können, müsste man freilich vorab eine breite Sichtung von Quellenmaterial verschiedener epistolographischer Zeiten und Phasen vornehmen. Erst dann ließen sich historische Konstanten und Spezifika ausmachen und konkretisieren.

Die eben aufgeworfenen Fragen kann und soll dieser Beitrag nicht umfassend beantworten. Vielmehr konzentrieren sich die Ausführungen auf Räume des ,empfindsamen' Briefes, auf Literaten wie Gleim, Ramler, Sulzer, Kleist oder Karsch und damit auf eine recht kurze zeitliche Spanne innerhalb der Briefgeschichte, die für die Entwicklung der Gattung Brief allerdings bekanntlich als zentral angesehen werden kann. ${ }^{14}$ Mit dem Garten, dem Kabinett und dem Schlachtfeld rücken sowohl tatsächliche Schreib- und Lektüreorte als auch Räume, über die zugleich ästhetische, literarische, soziale und politische Ansätze, Ideen und Vorstellungen transponiert und auch transferiert werden, in den Fokus. Epistolare Beziehungspflege und Distanzregulierung wird nicht selten über gemeinsame lebensweltliche

10 Vgl. u. a. Dünne u. Mahler 2015; Dünne u. Günzel 2006.

11 Vgl. Schuster 2014, 324-386.

12 Vgl. Bunzel 2008.

13 Vgl. Breuer 2019.

14 Vgl. zur empfindsamen Briefkultur u. a. Vellusig 2000; Vellusig 2011; Hentschel 2001; Wegmann 1988, 73-80; zur Epoche der Empfindsamkeit allgemein siehe u. a. Sauder 1974. 
und symbolische Räume praktiziert und etabliert. Dass der Garten in empfindsamen Briefwechseln eine oft nahezu ritualisierte Hintergrundszenerie darstellt und zugleich als wirkungsästhetischer und semantischer sowie als modellierter und inszenierter, mit Affekten und Emotionen aufgeladener Raum erscheint, ist bereits mehrfach thematisiert worden. ${ }^{15}$ Obwohl sich zahlreiche weitere Beispiele aus anderen Epochen anführen ließen, sind Gärten vor allem für die empfindsame Briefkultur und für die mit ihr verbundenen Reflexionen von Natürlichkeit, Emphase und Geselligkeit von besonderer Bedeutung gewesen. In deren epistolaren Eigenräumlichkeiten und Inszenierungsweisen geben sich sowohl neue Formen der Artikulation von Intimität als auch neue Lebens- und Liebeskonzepte zu erkennen, die sich nicht zuletzt als wesentlich für die Entwicklung kultureller Raumbildungsprozesse erweisen. Doch nicht nur der Garten und seine vielfältige epistolare Darstellung und Vergegenwärtigung, sondern auch das Kabinett (nicht selten als Gegenentwurf zum Garten) oder die Schlachtfelder der Kriege Friedrichs II. von Preußen erscheinen als zentrale lebensweltliche und epistolographische Räume. Letztere lösen bei empfindsamen Literaten wie Gleim, Ramler, Kleist, Lange oder Sulzer grenzen- und gattungsübergreifende Begeisterungsstürme aus, die sich neben Oden und Liedern auch in deren Briefwechseln niederschlagen und nicht zuletzt als elemantare Bestandteile dichterischer Gruppenbildungen und den damit verbundenen Verbalisierungs- und Abgrenzungsstrategien erscheinen, denen im Folgenden nachgegangen werden soll.

\section{Dialogische Wegeführung - Gärten und Briefe}

Dass der Garten als Artikulationsmedium für empfindsame Lese- und Schreibpraktiken von und in Briefen so an Bedeutung gewinnt, hat verschiedene Gründe. Gärten und Briefe bilden im Zeitalter der Empfindsamkeit nicht selten eine ästhetische und mediologische Einheit. Der Garten ist Inspirationsquelle, realer und virtueller Handlungs-, Begegnungs- und Rückzugsort sowie Schreib- und Leseraum. Er bietet - wie der Brief - Raum für vertrauliche Gespräche, Gefühlsschilderungen, gesellige Unterhaltungen, Projektionen, selbstreflexive Gedanken und emphatische Affekte und Gesten. Der Garten ist dabei vielfältiges Experimentierfeld und Bedeutungsträger. Er kann als Ort und Medium empfindsamer (geselliger oder einsamer) Naturbetrachtung und Naturwahrnehmung begriffen werden und wird damit zu einer Art Emotionslandschaft. Vor allem in dieser kognitiven Funktion scheint ihm innerhalb von Schreib- und Lektüreszenen des Briefes eine beson-

15 Vgl. u. a. Koebner 1977; Wegmann 1988, 90-97; Kittelmann 2020 b. 
dere Rolle zuzukommen. Die für die Mitte des 18. Jahrhunderts signifikante und von England durch die Schriften von Joseph Addison, dem Earl of Shaftesbury oder Alexander Pope und Gartenanlagen wie Stowe und Stourhead ausgehende Loslösung des Gartens von der barocken Regelgebundenheit korrespondiert mit den Briefstellern und Briefsammlungen der Zeit, die ebenfalls einen individuellen, einfachen, zwanglosen, intuitiven und natürlichen Briefstil propagieren. Wenn Joseph Addison im Spectator oder der in Berliner Gelehrtenkreisen um Johann Joachim Spalding, Karl Wilhelm Ramler und Johann Georg Sulzer intensiv rezipierte Earl of Shaftesbury darauf hindeuten, dass ihnen eine natürliche Wildnis lieber als alle barocke und geometrische Geziertheit sei, so lassen sich darin Parallelen zur zeitgenössischen Brieftheorie Christian Fürchtegott Gellerts oder Johann Wilhelm Ludwig Gleims erkennen. Seit den 1740er Jahren sagt eine junge Generation von Briefschreiberinnen und Briefschreibern sprachlichen Zwängen sowie barocken und servilen Titularien den Kampf an. Wie in der Gestaltung von Gärten, so kommt es auch in der Briefkultur zum Bruch mit tradierten, in diesem Falle rhetorischen Mustern. Die Gestaltungsprinzipien der neueren Landschaftsgärten stimmen in ihrem Zusammenspiel aus pittoresken Szenerien und literarischen Bezügen mit den Regeln des empfindsamen Briefstils überein. Wichtigstes Kriterium ist in beiden Fällen die (vermeintlich) natürliche Wiedergabe und Erweckung von Gefühlen. Folgt man diesem Anspruch in Gärten vor allem über Assoziationen und Gedankenverbindungen mit Hilfe von Naturkulissen, Architekturen, Gedenkurnen oder Schrifttafeln, so setzt auch der Brief in seinen intendierten Lektüreerlebnissen und -erfahrungen auf bestimmte Zeichen, Bildlichkeiten und Ikonographien des Natur- und Gartenraums. Die zahlreichen Einstreuungen von Begriffen wie Rasen, Hain, Quelle, blühend, Dickicht, Irrweg, Baum, Geißblatt oder Rosen in empfindsamen Briefwechseln und die Imagination akustischer Hintergründe wie singende Nachtigallen oder rauschende, fallende Blätter schaffen eine ästhetisch codierte Schreibumgebung der Verfasser, die sich auch auf die Lektüre des Gegenübers auswirken und einen gemeinsamen Raum bzw. virtuellen Treffpunkt schaffen soll. So schreibt Gleim an seinen Freund Ewald Christian von Kleist:

Ich habe einen kleinen Garten gekauft; den will ich für Sie zurecht machen, ich will Rasen für Sie darin zu sanftem Polster wachsen lassen, damit wir uns darauf lagern und den Nachtigallen zuhören können, die häufig kommen werden, meinem Kleist etwas zu singen. Kommen Sie nur zu mir!16

Bemerkenswert ist außerdem, dass der empfindsame Garten auf einem dialogischen Prinzip, auf dem Gespräch und der gemeinsamen Diskussion aufbaut. Die

16 Gleim an Ewald Christian von Kleist, 29. Dezember 1756, in: Kleist 1881, Bd. 3, 171. 
Wege in Landschaftsgärten sind meist so angelegt, dass zwei Personen nebeneinander gehen und sich austauschen können. ${ }^{17} \mathrm{Im}$ Brief trifft man eine ähnliche Konstellation an. Hier finden sich in VerfasserInnen und EmpfängerInnen ebenfalls mindestens zwei Personen zusammen, deren epistolarer Austausch zwischen Einsamkeit und Geselligkeit oszilliert. Der Garten wird in Briefen häufig als Ort der Zweisamkeit, der Gemeinschaft von Schreibenden und Lesenden imaginiert; wohl auch weil der „Verzeitlichungsmodus des Durchwanderns von Gärten“18 der schriftlichen, zeitlichen „Zerdehnung “19 in der epistolaren Kommunikation entgegenkommt.

Beispielsweise konzipiert und inszeniert Karl Wilhelm Ramler den brieflichen Dialog mit Gleim als gemeinsamen Spaziergang durch einen Garten:

Hier gehe ich oft gegen Untergang der Sonne in den Garten und nehme sie mit, und rede mit ihnen, und frage sie: aber sind sie mein ganz aufrichtiger Freund? nein ich frage sie so: glauben sie daß mein Hertz gantz aufrichtig sey? Dann sehen sie mich an und sagen ia, und schweigen und küßen. ${ }^{20}$

Dass das Anlegen von Gärten (nicht nur) unter Literaten in Mode kommt und innerhalb einer briefinternen topographischen Verortung an Bedeutung gewinnt, hat nicht zuletzt städtebauliche Gründe. Die Präsenz von Gärten in Korrespondenzen dokumentiert, wie eine zeitspezifische Raum- und Emotionskultur ineinandergreifen. So werden in der Mitte des 18. Jahrhunderts Gartenkultur und Gartenbau zunehmend als wesentlicher Teil der Landes- und Stadtverschönerung begriffen. Am Beispiel Berlins kann man sehen, wie im Zuge der Stadterweiterung mit der Anlage der Dorotheenstadt und der Friedrichstadt nicht nur den adligen Palais, sondern auch den Bürgerhäusern Gärten angeschlossen werden. Der Stadtplan des Grafen Samuel von Schmettau aus dem Jahr 1748 zeigt zahlreiche Obst- und Blumengärten, Taxusgärten, Orangerien und kleine Alleen, die zu Spaziergängen und geselligem Miteinander einladen sollen. Mit den öffentlichen und privaten Gärten entstehen im urbanen Raum neue Umgebungen, die wiederum die damaligen Lektüre-, Schreib- und Wahrnehmungspraktiken von und in Briefen beeinflussen. Berliner Dichter und Gelehrte wie Johann Georg Sulzer oder Karl Wilhelm Ramler legen sich Gärten an, arbeiten, lesen und schreiben (Briefe) darin. ${ }^{21}$ So präsentiert sich der in der Berliner Heiliggeiststraße situierte Garten des botanisch versierten Dichters Karl Wilhelm Ramler als bukolischer Ort mit Bezug auf

17 Vgl. dazu Verschragen 2001.

18 Niedermeier 2015, 325.

19 Ehlich 1993, 32.

20 Ramler an Gleim, 5. August 1746, in: Gleim u. Ramler 1906, 46.

21 Vgl. zu Sulzer auch van der Zande 1999. 
Theokrit, ${ }^{22}$ wenn Ramler seinem Freund Gleim von seinen „zweymal blühenden Aurikeln, wohlriechenden Levcojen und fabelhaften Narcißen und unglücklichen Hyacinthen“ berichtet und hinzusetzt: „Alle diese blühen schon in dem kleinen Garten; ich wolte die Rosen und Nelcken hinzusetzen, wenn sie, wie Polyphem im Theocritus scharfsinnig sagt, nur nicht etwas später blüheten. ${ }^{\text {“23 Und auch das }}$ mitten in Berlin gelegene Haus des Philosophen Johann Georg Sulzer stellt sich in zeitgenössischen Briefwechseln oft als ein von Najaden, Dryaden und Hirten bevölkertes urbanes Idyll dar, das zum geselligen Miteinander einlädt. Städtisches und ländliches Leben, gesellige und idyllisierende Praktiken, Authentiztität und Fiktion gehen in Orten wie diesem bzw. in dessen epistolarer Inszenierung eine Symbiose ein. Einem Brief Ramlers zufolge kann man dort „Bäume und Wiesen und Flüße und Gärten um sich haben, [sich] küßen und [die] Küße von den Najaden und Dryaden beneidet sehn." ${ }^{24}$ Sulzers Garten ist ein beliebter Treffpunkt für philosophische und gesellige Gespräche; das Interesse sowie die Teilnahme an der Entstehung des Areals insbesondere in Briefen von Freunden ist enorm: „Man trift unsern Sulzer jetzt niemals in vier Wänden an [...] er pflantzt Tannen für die Nachwelt, und Cichorienwurtzeln für seine Gäste“ "25 ist in einem Brief Ramlers zu lesen. Sulzer selbst will hier nichts Geringeres als den Garten des Epikur wiederherstellen und „die Musen in dem schatten der orangen Bäume [...] loken.“26 Dichterfreunde und -freundinnen fühlen sich zu poetischen Reflexionen angeregt und imaginieren sich in Sulzers Garten. In Anna Louisa Karschs Ode Sehnsucht nach der Freundschaft werden Sulzers junge Zypressenbäume besungen. Ebenso lobt Johann Jakob Bodmer, der selbst nie in Sulzers Garten gewesen ist und diesen allein in seiner dichterischen bzw. epistolaren Phantasie besucht, das Zusammenspiel von Stadt und Land, von Geselligkeit und idyllischer Natur: „Diesseits die heiteren Tage mit blumigten Düften [...]. Jenseits das offene Land mit grünen Tapeten. “27 In Bodmers Gedicht, das erst 1781 veröffentlicht wird, vollzieht sich sprachlich also die Verschmelzung von Natur (ländlich) und Stadt (Wohnung).

22 Bezüge auf antike Autoren finden sich ohnehin viele in den Korrespondenzen. So wird Horaz häufig zitiert. Ramler inszeniert sich in einem Brief, der einen gemeinsamen Spaziergang mit Sulzer nach Pankow zum Thema hat, als „Horaz der Panke“. Und Orte wie das Landgut von Horaz in den Sabiner Bergen, wo der Dichter bekanntlich ,alles aushalten und erdulden“ konnte, beeinflussen die Topographien und Raumimaginationen in empfindsamen Briefwechseln zweifelsohne nachhaltig. Vgl. Kittelmann 2020b.

23 Ramler an Gleim, 9. Mai 1755, in: Gleim u. Ramler 1907, 198

24 Ramler an Gleim, 28. April 1753, in: Gleim u. Ramler 1907, 21.

25 Ramler an Gleim, 20. Juni 1752, in: Gleim u. Ramler 1907, 352.

26 Sulzer an Bodmer, 30. März 1753, in: Sulzer u. Bodmer 2020, 244.

27 Bodmer 1781, 311. 
Im empfindsamen Brief sind die Grenzen gegenüber fiktiven Raumkonzepten und Topographien, etwa der zeitgenössischen Idyllendichtung, besonders offen und fließend. Die epistolare Artikulation und Inszenierung von Idylle ist vielfältig. ${ }^{28}$ Sie kann sich in der evozierten Umgebungssituation des Briefes widerspiegeln, etwa wenn Anna Louisa Karsch an Gleim schreibt, dass sie die Lektüre von dessen Briefen in einen „Hayn, am Rande des Waßers zum fuße der Mutter von Amor" verlagert habe. Die Lektüreszene in einem idyllischen Hain deutet auf die mythologische Überhöhung von Karschs Freundschaft mit Gleim hin, die in dem Wunsch mündet, dass Karsch sogar in Gleims Garten begraben werden und ihr ,Überrest [...] an dem Ort wo die Nachtigall nistet [...] zwischen zween Myrtenbaüme" seine letzte Ruhe finden soll. ${ }^{29}$ Zudem spiegelt sich die breit einsetzende Rezeption der Gattung Idylle in briefspezifischen Themen wie Natürlichkeit und Subjektvität wider. Natur wird dabei häufig als Flucht- und emotionaler Begegnungsraum evoziert, etwa wenn Sulzer dem befreundeten Schrifsteller Christian Fürchtegott Gellert seinen Garten als von der Brutalität und Aufgeregtheit der Welt abgeschirmten Ort darstellt:

Unsre Einsamkeit müßte Ihnen angenehm seyn. Es wartet ein Zimmer auf Sie, da Sie unter dem Gesang der Nachtigall einschlaffen und aufwachen können. Ich meine die freye, nicht die eingesperrte und ängstliche Nachtigall, die Sie in Leipzig genug hören können. Wir sind hier durch Gewäßer und durch cirkelnde Reyhen Hoher Bäume von der Bösen Welt abgesondert. ${ }^{30}$

Doch nicht nur Sulzers neo-epikureisches Gartenprojekt, sondern auch der Halberstädter Garten Gleims, der in der Tradition des griechischen Dichters Anakreon scherzhafte Lieder verfasst und ein weitverzweigtes freundschaftlichempfindsames Briefnetzwerk pflegt, markiert einen der zentralen Räume geselliger Kreise. In unzähligen Briefen beschreibt Gleim seinen Garten als bukolischen Ort und imaginiert sich und die Freundinnen und Freunde hinein. Dabei greifen ästhetische und naturkundliche Anverwandlungen von Natur durchaus ineinander und justieren epistolare Verfahren neu, etwa wenn man in den Briefen über konkrete Pflanzen, Bäume und Stauden ins Gespräch kommt. ${ }^{31}$ Gleims legendärer Halberstädter Kirschbaum und sein Walnussbaum, Sulzers Berliner Zypressen

28 Vgl. Kittelmann 2021.

29 Gleim u. Karsch 1997, 233.

30 Sulzer an Christian Fürchtegott Gellert, 7. Mai 1754, Biblioteka Jagiellońska, Kraków, Sammlung Autographa.

31 Diese Form der epistolar-geselligen Pflanzenverwendung erinnert an berühmte Idyllen wie Gessners Lycas, oder die Erfindung der Gärten, in der Nelken, Lilien, „Gloken-Blume“, „braune Scabiose“, Ulme, Winde, Rosen, Nelken, Klee, Geißblatt, Ysop und viele mehr sowohl feste Bestandteile als auch Akteure der idyllischen Szenerie sind. Vgl. Kittelmann 2021. 
oder Ramlers „duftende Levcojen“ sowie das Laublinger Blumenbeet von Anna Dorothea Lange avancieren zu einem verbindlichen und gemeinschaftsstiftenden idyllischen Inventar, das in Briefen fast ritualisiert sowohl als Hintergrundszenerien als auch als wirkungsästhetischer Bedeutungsträger eingesetzt wird und zugleich zentrale Räume der Empfindsamkeit mitkonstituiert.

\section{Brisanz und Intimität}

Empfindsame Dichterkreise und BriefschreiberInnen finden im Garten und dem ihm inhärenten Spannungsfeld aus Authentizität und Literarizität, Intimität und (Brief-)Öffentlichkeit, bukolischer Szenerie und idyllischer Naturwahrnehmung eine ideale Imaginationsfläche ihres vornehmlich in Briefen kultivierten Geselligkeits- und Freundschaftsideals. Nicht zuletzt erscheinen der Garten und die in ihm situierten Praktiken, Schreib- und Leseszenen ${ }^{32}$ als ein Ort der Geschlechterspannung, als Projektionsraum sinnlicher Erfahrung und möglicher (erotischer) Entgrenzung. Hier sind sowohl ein geschlechterübergreifendes und gefühlsbetontes Geselligkeits-, Gemeinschafts- und Freundschaftsideal als auch gesellige Gesten und Affekte wie Scherzen, Weinen, Küssen und andere Zärtlichkeitsbekundungen möglich, die im Innenraum, in der Stube oder im Kabinett nur bedingt realisierbar sind, ja sogar als unschicklich und brisant angesehen werden. Während eines Berlin-Besuches ist Gleim vom Tiergarten auch deshalb so fasziniert, weil man nicht nur ,auf der Spree mit den Schwänen um die Wette [...] schwimmen“, sondern „sich mit der gantzen Gesellschaft unter tausend Mädchen verirren" konnte. ${ }^{33}$ Obgleich sich Briefschreiberinnen erstaunlich oft am Schreibpult mit der Feder in der Hand inszenieren, ${ }^{34}$ werden sie von ihren männlichen Korrespondenz- oder Lebenspartnern oft im Garten gedacht und/oder dort die gemeinsame Begegnung imaginiert. „Was thust du denn den ganzen Tag? Bist du allein im Garten, oder ist noch jemand da? Was liesest Du?" fragt etwa Johann Georg Sulzer seine Braut Wilhelmine Keusenhoff, die sich zu diesem Zeitpunkt in dem auf einer Halbinsel in der Elbe gelegenen Garten von Heinrich Wilhelm Bachmann aufhielt. „[I]ch irre auf der gantzen Insel herum, dann nehme ich mahl

32 Zum Begriff der „Schreibszene“, der von Rüdiger Campe eingeführt und etabliert wurde, vgl. Campe 1991.

33 Gleim an Uz, 16. August 1758, in: Gleim u. Uz 1899, 291.

34 Vgl. etwa Briefe und Porträts von Sophie von La Roche, Charlotte Schiller oder Anna Louisa Karsch. Zur Bedeutung des Gartens als Handlungsort bei Sophie von La Roche vgl. allerdings Roßbach 2015, 202-205. 
den Haller“, ${ }^{35}$ bemerkt sie zurückhaltend und entwirft in ihrem Brief doch zugleich bekannte Szenerien einsamer Liebender in unberührter Landschaft, wie sie etwa in Hallers Ode Doris oder in zeitgenössischen Kupferstichen ${ }^{36} \mathrm{zu}$ finden sind.

Im selben Garten weilt einige Zeit später Klopstock, der hier am Messias schreibt und Maria Sophia Schmidt, seiner „Fanny“, berichtet, dass er nachts um zwölf Uhr aufgestanden und allein im „Garten herumgegangen“ sei, an sie gedacht und den Brief verfasst habe: „Eine wahrhaftig himlische Stunde.“" ${ }^{37}$ Über den Ort des Schreibens und der Lektüre wird nicht zuletzt das im Brief Unsagbare wie erotisches Begehren oder voreheliche Sexualität thematisiert. Nicht selten avanciert der Garten als Lektüre- und Schreibraum zu einem Sehnsuchts- und Erinnerungsraum an die entfernte Freundin oder Geliebte. Brief und Garten verbinden sich zu einer geistig-emotionalen Landschaft, in die man sich hineinschreiben, in der man frei von Koketterie und Zwängen agieren und sich ganz von seinen Gefühlen leiten lassen kann, wie wiederum ein Brief Sulzers zeigt:

\footnotetext{
Ich komme voll Schweiß, sehr müde und meiner Gewohnheit nach sehr melancholisch von dem Thiergarten in meine einsame und traurige Kammer zurük. [...] Als ich diesen Abend von der Academie kam, fühlte ich einen solchen Wiederwillen nach Hause zu gehen, daß ich mich in Gedanken immer mehr von zu Haus entfernte, bis ich endlich in den Park kam. Ich irrte einsam aus einer Allee zur andern; ich wollte mich recht aufmuntern, aber jedes Blatt schien mir deinen süßen Nahmen ins Ohr zu säuseln. Ich antwortete Ihnen durch tieffe Seüfzer. Eine Allee führt mich hinaus auf das freye Feld, wo ich die Sonne untergehen sah. Ich sah nicht auf die Sonne, sondern nach der Gegend, die mein liebstes hält. ${ }^{38}$
}

Brief und Raum sind hier in dynamischer Weise miteinander verbunden: durch bestimmte Lemmata und Wortwendungen wie „fühlen“, „irren“, „sehen“ oder „seufzen“ wird die Geliebte über akustische und visuelle Signale anwesend gemacht. Über den epistolaren und topographischen Raum treten hier der Ort des Briefschreibers (Berliner Tiergarten) und der Ort der Lesenden (Bachmanns Garten in Magdeburg) in Verbindung zueinander und ermöglichen die Vereinigung im Brief.

Sulzers vornehmlich im Jahr 1750 entstandener Briefwechsel mit seiner Braut und späteren Ehefrau Wilhelmine Keusenhoff dokumentiert sowohl die Schnittstelle zwischen realen und imaginierten Orten als auch die Dynamiken privater

35 Wilhelmine Keusenhoff an Sulzer, 9. Juni 1751, zit. nach Kittelmann 2019, 78.

36 Vgl. zum Beispiel Daniel Chodowieckis Kupferstich Die Unterredung, der im Göttinger „TaschenCalender" für das Jahr 1779 abgebildet ist.

37 Klopstock 1979, 104.

38 Sulzer an Wilhelmine Keusenhoff, [Juni 1757], zit. nach Kittelmann 2019, 79. 
und intimer Räume und deren epistolare Existenz. ${ }^{39}$ Was im Garten möglich war, musste im Kabinett zuweilen ungesagt bleiben. ${ }^{40}$ So ist sich Sulzer der Brisanz seiner Zeilen an seine Braut Wilhelmine, der er den zukünftigen gemeinsamen Schlafplatz entwirft, durchaus bewusst:

Sie werden sich beqüemen müßen in eben diesem Alcove zu schlaffen. Aber wie wird es sich schiken, daß Sie bey einer Mansperson in einem Zimmer schlaffen? Was wird man wol von Ihnen urtheilen? Wird man nicht denken wir .... Aber man muß die Leüthe reden laßen. [...] Könnte ich nur schon in den Alcoven gehen, da Sie schlaffen werden. Wie hurtig wollte ich das Schreiben laßen um zu bette zu gehen? Aber mein einsames bette hat nichts das mich dahin lokt. Doch ja es hat auch was. Es flattern darin angenehme und süße Träume herum. Diese laßen mir ofte meine allerliebste Freundin sehen und ofte laßen Sie mich dieselbe küßen. Ich will hurtig gehen von diesen Träumen mich täuschen zu laßen. ${ }^{41}$

Das Unsagbare, die sexuelle Intimität der Brautleute, wird hier sowohl über vier Auslassungspunkte und somit über eine non-verbale Kommunikation als auch über Worte und räumliches Interieur wie „schlaffen“, „in einem Zimmer“ oder „Bette“ evoziert. Die Antwort Wilhelmines, so es sie denn überhaupt gegeben hat, ist nicht überliefert. Mit der Eheschließung einige Wochen später verliert der Raum, auch im Brief, ohnehin an jeglicher Brisanz und wird nun als Ort bürgerlichfamiliärer Privatheit stilisiert, wie ein Schreiben Sulzers an seinen engen Freund, den Winterthurer Rektor Martin Künzli, zeigt:

Ich habe nun meine kleine angenehme Familie so nahe um mich, als möglich ist, in dem Zimmer wo wir speißten, da Sie bey uns waren, ist meine Willhelmine, und in der Stube, die zwischen meiner und ihrer ist, wohnt unsre Jugend, so daß wir alles hören was bey dieser vorgeht, und diese Einrichtung sezet mich außer Sorgen, daß diese Kleinen durch ihre Aufwärterinnen verdorben werden. Dieses macht nun zwahr, daß ich oft in meinen Verrichtungen gestört werde, insonderheit, da die Mine sehr oft anklopft um zu mir zu kommen, aber ich habe selten wichtigere Verrichtungen, als die sind, die zur Erziehung gehören. ${ }^{42}$

Künzli, der während eines Besuches in Berlin von Mitte August bis zum 23. September 1753 bei Sulzer wohnt, wird hier nun über die Räumlichkeiten in das Sulzer'sche Familienleben einbezogen. Künzlis Kenntnis der privaten Räume wird gezielt angesprochen (,wo wir speißten“) und über diese gemeinsame Erinnerung epistolare Vertrautheit hergestellt. Zudem war Künzli nicht nur mithilfe von Briefen, sondern

39 Vgl. Kittelmann 2019.

40 Vgl. dazu auch Lehnert 2014.

41 Sulzer an Keusenhoff, [September 1750], zit. nach Kittelmann 2019, 77.

42 Sulzer an Martin Künzli, 1. März 1754, Studienbibliothek/Winterthurer Bibliotheken, Sign. Ms BRH 512/72. 
auch mit einem Porträt permanenter Gast in Sulzers Haus. Sulzer hatte für die Porträts seiner engsten Freunde und Vertrauten extra „einen Plaz [...] ausersehen, den sie zieren sollen [...] eine schöne und sehr angenehme StudierStube. “43 Solche Freundschaftskabinette oder Freundschaftsgalerien ${ }^{44}$ zierten viele Privat- und Dichterhäuser. Die bekannteste ist wohl die Freundschaftsgalerie von Johann Wilhelm Ludwig Gleim, die nahezu komplett im Halberstädter Wohnhaus des Dichters erhalten und überliefert ist. ${ }^{45}$ Gleim lässt seine Freunde in Berlin und anderswo per Brief an der Gestaltung teilhaben. Das reicht bis hin zur Auswahl und detallierten Beschreibung der Tapeten, ${ }^{46}$ die sein Haus zieren sollen:

Der unartige und liebe Hempel, ${ }^{47}$ was macht doch der? Er will also nicht wieder zu mir kommen. Thut er es dis Jahr nicht, aufs Jahr soll er gewiß, denn dann soll er mir nur eine Blume in mein Cabinet der Freundschaft mahlen. Ich laße mein Hauß bauen, da soll er mir in drey Zimmer Tapeten schaffen, das sagen sie ihm nur bey Zeiten. Halten sie nur auch hübsch ihr Versprechen und treiben ihn an, daß er mir die Köpfe meiner Freunde bald fertig schaft. Ist er mit dem ihrigen schon fertig? O schicken sie mir ihn doch. ${ }^{48}$

Sowohl in den privaten Porträtgalerien als auch in der epistolaren Imagination gemeinsamer Zusammenkünfte offenbart sich eine medial vielfältige Semantisierung von Freundschaft und Geselligkeit und damit verbunden der permanente Versuch der Regulierung und Überbrückung von Distanz im privaten Raum. Wenn man, wie im Falle Ramlers und Gleims räumlich getrennt war, blieb oft ,nur` die epistolare Kommunikation, bei der man sich das Gegenüber im eigenen Zimmer dachte und dort mit ihm ins Gespräch kam:

Ich kan nicht lange sitzen, ich gehe von einem Zimmer ins andre. Da kommt mein Gleim, und fällt mir um den Hals - Ich kan nicht weiter; ich bin zärtlich. Sehen Sie, mein liebster Gleim, so unterhalte ich mich mit meinen Gedancken, wenn ich auch nicht einsam bin. Das Schicksal kan mir so viel Freude nicht verderben, ich muß sie sehen, ich muß sie in ihrer ruhigen Heymath sehen, und ich muß sie allein genießen. ${ }^{49}$

43 Sulzer an Martin Künzli, 15. November 1750, Studienbibliothek/Winterthurer Bibliotheken, Sign. Ms BRH 512/72.

44 Vgl. dazu auch den Beitrag von Claudia Bamberg in diesem Band.

45 Vgl. Scholke u. Adam 2000.

46 Vgl. dazu auch Pott 2013; Holm 2018.

47 Gemeint ist der Maler Gottfried Hempel.

48 Gleim an Ramler, 15. August 1751, in: Gleim u. Ramler 1906, 302. Ramler antwortet: „Hempel will jetzt eine jede Pause, die er machen darf, auf ihr Porträt wenden und des Abends den Riß fertig machen. Meßen sie doch die Höhe und die Breite ihrer großen Wand und auch den Raum bey den vier Fenstern und den beyden Thüren und schicken mir diese Nachricht mit der ersten Post.“ (Gleim u. Ramler 1907, 10).

49 Ramler an Gleim, 20. Juni 1751, in: Gleim u. Ramler 1906, 351. 
In solchen brieflichen Äußerungen gibt sich der Drang zu erkennen, auf dem Papier reale Räume der Begegnung aufzusuchen bzw. zu schaffen. Der Brief als Schreibund Denkort und das Kabinett als realer Raum, wo nicht nur das Schreibepult steht oder der Alltag absolviert wird, sondern auch Geselligkeit und exklusive Freundschaft ihren Platz haben, bilden eine mediale Einheit, eine Art exklusive Topographie der Freundschaft.

Zudem können der Fortgang eines Briefes und die Beschaffenheit des Zimmers, in dem er verfasst wird, unmittelbar zusammenhängen, etwa wenn Ramler einen Brief an Gleim mit den Worten schließt: „Mein Zimmer ist kalt und mein Caffe aus. Schlafen sie wohl“"50 oder wenn ein über den Bruch der Freundschaft mit Klopstock verzweifelter Bodmer schreibt: „Ich habe sonntags und montags in meinem Closet die thränen der zärtlichen Wehmut aus den Augen, und nicht bloß aus der feder geweint." ${ }^{51}$ Die Beziehung zwischen epistolarem und realem Raum sind durchaus fragil. Nicht selten wirkt sich die Umgebung störend und beeinträchtigend aus, etwa wenn Wilhelmine Sulzer ihrem Mann berichtet, dass sie der „Lärm auf der Strasse“ 52 ablenkt oder wenn dieser in einer Wirtshausstube einfach nicht zum Schreiben kommt und schließlich ungewollt die Worte fremder Menschen Eingang in seinen Brief finden:

Ich size in einem kleinen Stübchen unter hundert Leuten, die mir alle miteinander unbekannt sind. Neben mir sizen drey Frauenzimmer die mit vielem Schwazen mich immer stöhren und einige Schritte von mir sind zwanzig Musicanten, davon ein jeder sein Instrument stimmt. Ein abscheüliches Gelaerm. Bey dem allen aber fühlt mein Herze gar nichts, als nur die Zärtlichkeit für Sie, mein allerliebstes Kind, dies ist alles was ich Ihnen sagen kann. Tragen Sie nur Geduld bis den andern Morgen, als dann werden Sie einen weit größern Brief von mir empfangen. Denn so gern ich izt auch diesen anfüllte, so ist mir doch unmöglich bey einem solchen Laermen zu schreiben, ohne tausend mal zu verirren. Gehorsame Dienerin Fr. Baron, wie befinden Sie sich. Sind Sie vergnügt gewesen, die Zeit über da wir das Vergnügen nicht gehabt haben Sie zu sehen. [Hervorh. JK] Sehen Sie ich schreibe schon nach was man neben mir spricht. Izo geht die Musik an. ${ }^{53}$

Das Schreiben in der Öffentlichkeit, in geselligen und ,riskanten` Räumen wie dem Wirtshaus, ${ }^{54}$ gestaltete sich als schwierig. Das intime und emotionale Gespräch verlangte vielmehr den Rückzug ins Kabinett, ja in einigen Fällen sogar in das vom geselligen Treiben abgeschirmte Bad, wie ein während eines Besuches in der

50 Ramler an Gleim, 4. März 1748, in: Gleim u. Ramler 1906, 103.

51 Bodmer an Sulzer, Februar 1751, in: Sulzer u. Bodmer 2020, 170.

52 Keusenhoff, an Sulzer, 10. Mai 1756, zit. nach Kittelmann 2019, 76.

53 Sulzer an Keusenhoff, 26. September 1750, zit. nach Kittelmann 2019, 76.

54 Zum Wirtshaus als literarischem und zugleich prominentem Ort des Ausnahmezustandes vgl. Neumeyer 2015. 
Schweiz verfasster Brief Sulzers an seine Braut Wilhelmine offenbart, der wiederum zugleich mit einer ironischen Reminiszenz an rokokohafte Szenerien spielt:

D'ailleurs je vous ecris ceci dans une attitude fort singuliere. Je suis dans l'eau, presque jusqu'au menton. C'est dans un bain que j'ecris, c'est le seul moment, ou je suis à moi depuis mon arrivée. Et j'ai expressement dit à toute la compagnie, que je m’y mettrai tout nud, pour les empecher de m'interrompre, en vous ecrivant. Vous riries bien si vous me voyiés maintenant. Figurés vous ces divinités des fleuves et des fontaines, qu'on peint dans dès grottes au millieu des eaux. Je leur ressemble un peu asteur. ${ }^{55}$

Der Rückzug ins Kabinett ist in empfindsamen Kreisen nicht selten gleichbedeutend mit dem (freilich oft inszenierten) Rückzug in den privaten Brief, in die intime Artikulation von Freundschaft und damit verbunden in die eigenen Gefühle und Empfindungen bzw. deren epistolare Reflexion. Während der Garten häufig für eine Entgrenzung im erotischen, affektreichen und sinnlichen Sinne steht, ist das Kabinett mit einer bewussten Entgrenzung und Inszenierung freundschaftlicher Gefühle verbunden. Hier muss man sich nicht davor „scheühen“, wie in einem Brief Sulzers an Bodmer zu lesen ist, im „Schlaffrok und in der Nachtmüze“ ${ }^{\text {"56 }} \mathrm{zu}$ erscheinen, sondern im Gegenteil: gerade hier bietet sich ein vor dem öffentlichen Zugriff sicherer Raum, ein Rückzugsort, der zugleich der Verfestigung von Freundschaft und der gemeinsamen Identitätsstiftung dienen kann. Empfindsame Briefe dienen der Kartographierung des eigenen Innenlebens und der geselligen Reflexion; brauchen allerdings zugleich eine konkrete topographische Verortung, wie ein Brief Sulzers an Gleim deutlich vor Augen führt:

Ich stelle mir schon im Geist das Vergnügen vor so wir haben würden, wann wir sie von hieraus besuchen würden. An mir soll es nicht fehlen. Ich habe nicht gern einen an einem Orte den ich nicht kenne. Ich muß mir ihn in seiner Stube in seiner Kammer, an seinem Fenster u. s. f. vorstellen können, sonst kömmt es mir vor, als wenn er in einem dunkeln Loch wohne, an welches ich nicht gern denke. ${ }^{57}$

Die in empfindsamen Briefwechseln häufiger zu findenden Wendungen „mein Zimmer“, „mein Cabinet“ oder „mein Closet“ stehen sowohl für eine zeittypische

55 Sulzer an Wilhelmine Keusenhoff, 24. Juli 1750, Freies Deutsches Hochstift - Frankfurt a. M. Sign. Hs. 2999. Übers.: „Und ich habe der ganzen Gesellschaft ausdrücklich gesagt, dass ich mich ganz nackt ins Bad setzen werde, um zu verhindern, dass sie mich beim Schreiben unterbrechen. Sie würden sehr lachen, wenn Sie mich jetzt sähen. Stellen Sie sich diese Fluss- und Brunnengottheiten vor, die in Grotten im Wasser gemalt sind. Diesen bin ich im Moment ein bißchen ähnlich." Für die Unterstützung bei Transkription und Übersetzung danke ich Baptiste Baumann.

56 Sulzer an Bodmer, 9. September 1774, in: Sulzer u. Bodmer 2020, 929.

57 Sulzer an Gleim, 3. Februar 1748, Museum der deutschen Aufklärung - Gleimhaus Halberstadt, Hs. A 4072. 
Individualisierung als auch für die Möglichkeiten einer exklusiven Gruppenbildung, etwa wenn man bewusst entscheidet, mit wem man in einen intimen Briefwechsel tritt und wen man damit in das eigene Zimmer hineinlässt. So bemerkt Sulzer in einem Brief an Gleim, dass er die „beste Stube“ für seine „auswärtige Freünde“ reserviert habe und fordert ihn mit dem gleichen Federstrich auf, diese „einzuweyhen“: „Sie [die Stube, Anm. JK] verdienet, daß dieses von einem Poeten geschiehet." $" 58$

Dass die gemeinsame topographische Verankerung und die (epistolare) Schaffung kollektiver Räume für die empfindsame Briefkultur und deren Akteurinnen und Akteure zentral ist, zeigen nicht nur die eben ausgeführten Beispiele zum Garten und Kabinett, sondern auch die epistolare Präsenz des Schlachtfeldes, obgleich es zunächst kaum etwas mit der intim-konzentrischen Abgeschlossenheit des Gartens oder Kabinetts gemeinsam zu haben und vielmehr in einem drastischen Kontrast dazu zu stehen scheint. Tatsächlich eröffnet der Blick in die Briefwechsel empfindsamer Autoren wie Gleim, Ramler, Kleist oder Sulzer eine auffällige Präsenz von Kriegsschauplätzen und Schlachtfeldern, die sowohl als Schreibeort als auch als imaginierte und mit einer klaren politischen Intention beschriebene Räume bedeutsam sind.

\section{Mars und Musen - Epistolare Schlachtfelder}

Dass ein „Officier, der unaufhörlich prügeln muß,59 zugleich „Garten-Idylle[n] in abscheulichem Tumult und Unruhe" ${ }^{60}$ verfassen und somit ein empfindsamer Dichter auch ein Soldat und Militär sein kann, zeigt das Beispiel Ewald Christian von Kleists, der eng mit Gleim, Ramler, Sulzer und Salomon Gessner befreundet ist und mit dem empfindsamen Gedichtzyklus Der Frühling europaweit bekannt wird. Als Angehöriger der preußischen Armee und Kompaniechef ist Kleist aktiv in Kriegshandlungen des Siebenjähriges Krieges involviert und mit Sterben und Tod konfrontiert. Die expansive, brutale und globale Umgebung, in der er als Militär agiert, hat so gar nichts mit den in seinen Gedichten und Briefwechseln evozierten geselligen Idyllen, wo man sich in einen „Irrgarten wie Schäfer“ legen und „die Milch von unsern Schafen“61 trinken will, gemeinsam. Kleists grausame Ver-

58 Sulzer an Gleim, 12. Dezember 1752, Museum der deutschen Aufklärung - Gleimhaus Halberstadt, Hs. A 4126.

59 Ramler an Gleim, 15. Oktober 1746, in: Gleim u. Ramler 1906, 60.

60 Kleist an Gleim, 4. Dezember 1757, in: Kleist 1881, Bd. 2, 457.

61 Ramler an Gleim, 19. Juni 1748, in: Gleim u. Ramler 1906, 133. 
wundung auf dem Schlachtfeld von Kunersdorf und sein Tod wenige Tage später erschüttern seinen Freundeskreis dementsprechend nachhaltig und werden in unzähligen Briefen diskutiert und betrauert. Viele der Briefschreiber und Briefschreiberinnen stellen sich zwar nicht militärisch wie Kleist, aber doch poetisch mit Oden und Liedern in den Dienst Friedrichs II. von Preußen und seiner Kriege. ${ }^{62}$ Gleim, das Ehepaar Samuel Gotthold Lange ${ }^{63}$ und Anna Dorothea Lange - die schon 1745 während des Zweiten Schlesischen Krieges für den ,göttlichen Vater Friedrich“ ihr „Blut versprützen“64 will -, Ramler sowie in späterer Zeit Christian Felix Weiße, Friedrich Wilhelm Gerstenberg und Anna Louisa Karsch besetzen und modellieren eine noch nicht vollends ausgestaltete Gattung patriotischer Lyrik mit Elementen einer bis dato eher der Ependichtung vorbehaltenen nationalen Poesie, wie etwa dem Herrscherlob, der Besingung errungener Siege oder der Beschreibung nationaler Tugenden. Zugleich zeugen sowohl private als auch publizierte Briefe nachdrücklich von einer Mobilisierung für Friedrich II. und Preußen. Das Interesse an Friedrich, vor allem auch an seiner Person, seinem Leben, seinem Tagesablauf ist enorm. Briefe wie die Lettres familiaires des Vertrauten Friedrichs, Jakob von Bielfeld, die 1763 erschienen und Einblicke in das Privatleben des Königs gaben, erfreuen sich großer Beliebtheit und deuten auf die Entstehung eines wahren Briefkultes um Friedrich hin. Die publizistische Selbstinszenierung und philosophische Erfindung Friedrichs und des noch jungen Königreichs Preußen findet zweifelsohne eine Analogie in Briefwechseln der Zeit. ${ }^{65}$

Anhand der Präsenz Friedrichs II. und seiner militärischen Aktionen zeigt sich die Verschränkung von Historie und epistolarer Kommunikation bzw. der Einfluss, den historische Begebenheiten sowohl auf Themen als auch auf die Verfasstheit, Gestaltung, emotionalen Affekte und nicht zuletzt die evozierten Räume von Briefen haben. Briefe avancieren dabei zu Schnittstellen zwischen der militärischen und der lebensweltlich-literarischen Sphäre. Das lässt sich bereits

62 Vgl. Hildebrandt 2019.

63 Lange, der in seinen Horatzischen Oden (1745) Friedrichs Siege besingt, gehört zu den wenigen, die die Aufmerksamkeit Friedrichs, der der deutschen Literatur bekanntlich skeptisch bis ablehnend gegenübersteht, erregen und sogar ein Dankesschreiben von ihm erhält.

64 Lange 1745, 166.

65 Gustav Volz stellt 1926 u. 1927 eine Anthologie mit dem Titel Friedrich der Große im Spiegel seiner Zeit zusammen, die die Biographie, den Charakter und die Besonderheit des Königs anhand von Briefen präsentieren soll. Er versammelt hier in drei Bänden zahlreiche Briefe, u. a. von Gleim, Ramler, Sulzer, Bodmer, Voltaire, D’Alembert und Militärs wie Jakob Keith. Volz stellte die Briefe allerdings ausschließlich auf Deutsch bzw. in deutscher Übersetzung vor. Ein wesentlicher Aspekt ging dabei verloren: die epistolare Kommunikation über Friedrich ist von Beginn an polyglott. Man tauscht sich auf Französisch, auf Deutsch, auf Englisch und in anderen Sprachen über den König aus. 
anhand der Datierung zahlreicher Briefe Kleists beobachten, die mit Beginn des Siebenjährigen Krieges im Jahr 1756 häufig aus Winter- oder Sommerquartieren wie dem „Lager bei Maxen“, dem „Lager bei Dippoldiswalde“ oder „Auf dem Schlachtfelde bei Borne, abends den 5. Nov. 1757“ geschrieben und versendet werden; etwa an Gleim, anakreontischer „Amor im Harnisch“, ${ }^{66}$ der mit seiner 1758 erschienenen Sammlung der Preussischen Kriegslieder in den Feldzügen 1756 und 1757 für Furore sorgt und für jede Informationen von den Schlachtfeldern, die anschließend in seinen Briefnetzwerken Verbreitung finden, dankbar ist:

Indeß, da es nicht anders sein kann, so will ich mich so lange über unsere Siege freuen, als Sie, mein liebster Kleist, mir solche erzählen. Sie haben es diesmal so ausführlich und so deutlich gethan, daß man das Schlachtfeld vor sich sieht und sich fürchtet, noch nicht sicher darauf zu sein. Ihr Schreiben ist schon hundertmal abgeschrieben, ohngeachtet es erst heut Nachmittag um 4 Uhr ankam; ich habe es schon so oft vorgelesen, daß ich es auswendig kann, und ich kann

Der König lebt, zehntausend Feinde sind todt.

Der Enkel, Sieger Friederich,

Glaubt Deine Wunder nicht;

Sie lesend, überzeugt er sich,

Er les’ ein schön Gedicht. ${ }^{67}$

Die Ereignisse des Siebenjährigen Krieges führen zu einer Flut an Schlachtbeschreibungen, Friedrich-Anekdoten und patriotischen Bekenntnissen innerhalb von Korrespondenzen ${ }^{68}$ weit über Preußen hinaus, wie der Briefwechsel zwischen dem in Berlin lebenden Schweizer Johann Georg Sulzer und seinem Zürcher Landsmann Johann Jakob Bodmer zeigt. Bodmer, der gemeinsam mit dem Arzt Laurenz Zellweger in Trogen und Martin Künzli in Winterthur einen wahren und dennoch für die republikanischen Schweizer nicht unbedingt selbstverständlichen Kult um Friedrich II. zelebriert, giert geradezu nach Nachrichten und Informationen über den großen König, den „preussischen Helden“ und „neües aus dem Felde““. ${ }^{69}$ Der mit Generälen wie Heinrich Adrian von Borcke eng befreundete Sulzer kann diese fast direkt vom Schlachtfeld liefern. Mehrfach bittet Sulzer Borcke um Auskünfte, die er dann in seine privaten Korrespondenzen einfließen lässt. Wichtig sind dabei

66 Lee 2011. Für Gleim fand der Krieg vornehmlich auf dem Papier statt. Er besuchte Kleist nur einmal, als dieser 1757 mit der preußischen Armee vor Leipzig stand, und schrieb im Anschluss: „[J]etzt träume ich alle Nacht von meinem Aufenthalte bei Ihnen; ich fahre mit Ihnen spazieren, ich bin mit Ihnen in der Schlacht und fasse einen Panduren beim Bart und höre Sie sagen: ,Seht mir doch da, den Gleim!““ (Kleist 1881, 194).

67 Gleim an Kleist, 12. Dezember 1757, in: Kleist 1881, Bd. 3, 151.

68 Vgl. dazu u. a. Lacher 2017; Disselkamp 2005; Décultot u. Kittelmann 2020, XXVIII-XXXIII.

69 Bodmer an Sulzer, 8. Januar 1757, in: Sulzer u. Bodmer 2020, 334. 
vor allem exakte Lagepläne und eine genaue Wiedergabe der topographischen Situation, wie der folgende Brief verdeutlicht:

\begin{abstract}
Monsieur. Quoique je sache fort bien, qu'il convient aux Muses d'être retirées pendant que Mars tirannise la terre, j'ose pourtant vous envoyer une lettre, jusqu'au sein même d'une Armée. Comme Vous n'êtes pas tout à fait guerrier et que ma Muse ne se pique pas justement de chasteté, elle m’a pressé depuis longtems de Vous écrire. J'espère que Vous mettréz à profit la tranquillité des quartiers d'hiver et l'opportunité de Vous trouver au millieu d'une petite Armée, pour Vous informer de tout ce qui regarde les exploits de nos heros pendant la campagne passée. Nous savons bien en gros ce qu'ils ont fait, et nous avons même assez de jugement pour prévoir toujours qu'ils feront de grandes choses, et qu'ils vaincront tout énnemi. Mais Vous savez bien, que cette theorie est un peu sterile, à moins qu'on ne sache toutes les circonstances des choses. Vous êtes sans doute en état de Vous procurer des plans exacts des terrains, et de savoir par des bouches veridiques des anecdotes, qui caracterisent mieux les actions, que les relations generales. Nous venons de récevoir une relation abregée de la campagne, qui paroit d'une plume du premier rang. Mais cela est si honteusement defiguré par l'impression, et d'ailleurs d'un stile si concis, que notre cupidité de savoir tous les exploits de nos heros, en est plutôt irritée, que rassasiée. ${ }^{70}$
\end{abstract}

Obwohl der Antwortbrief Borckes nicht überliefert ist, lassen Sulzers Briefe darauf schließen, dass ihn die gewünschten Informationen erreichten. In seiner Korrespondenz mit Bodmer finden sich seitenlange Ausführungen zur Bewegung und Stellung der Truppen, zu großen Schlachten und kleineren Scharmützeln und vor allem zu den landschaftlichen Gegebenheiten. Einzelne Ortschaften, Wälder und Flüsse werden akribisch verzeichnet. Die Topographie und Ikonographie des Krieges werden in die Topographie und Ikonographie der Briefe und damit in das

70 Sulzer an Heinrich Adrian von Borcke, 1. Dezember 1756. Zentralbibliothek Zürich, MS Briefe Sulzer. Zit. nach Sulzer u. Bodmer 2020, 1251. Übers.: „Mein Herr, obgleich ich sehr gut weiß, dass es den Musen ziemt, sich zurückzuziehen, wenn Mars die Erde tyrannisiert, dennoch wage ich es, Ihnen einen Brief bis in die Mitte einer Armee zu schicken. Da Sie nicht ganz ein Krieger sind und meine Muse nicht gerade auf Unberührtheit besteht, hat sie mich seit Langem gedrängt, Ihnen zu schreiben. Ich hoffe, dass Sie von der Ruhe der Winterquartiere und dem Vorteil, inmitten einer kleinen Armee zu sein, profitieren werden, um sich über alles zu erkundigen, was die Taten unserer Helden während des vergangenen Feldzuges betrifft. Wir wissen wohl im Ganzen, was sie getan haben, und wir besitzen sogar Verstand genug, um vorauszusehen, dass sie Großes vollbringen und jeden Feind besiegen werden. Doch Sie wissen ja, dass diese Theorie etwas fruchtlos ist, es sei denn, man wüsste alle Umstände. Sie sind zweifellos imstande, exakte Lagepläne zu besorgen und von vertrauenswürdigen Zungen Anekdoten zu kennen, die die Handlungen besser charakterisieren als die allgemeinen Berichte. Wir haben jetzt einen verkürzten Bericht des Feldzuges erhalten, der von einer erstrangigen Feder zu stammen scheint. Aber durch den Druck wurde er so schändlich entstellt, und dazu ist er so knapp, dass unsere Begierde, alle großen Taten unserer Helden zu kennen, viel eher gereizt als gesättigt wurde.“ 
Bewusstein ihres Verfassers und Adressaten geradezu überführt. ${ }^{71}$ In ihrer Bildlichkeit und Anschaulichkeit erinnern Sulzers Briefe an zeitgenössische Bildmedien wie etwa Johann Martin Bernigeroths Kupferstichserie Neues Kriegs-Theater.

Die Darstellungen sind so genau, dass sich Bodmer dazu aufgerufen und berufen fühlt, Oden und Hexameter auf die Schlacht bei Planian, bei Roßbach oder auf die Oder als Fluss militärischer Auseinandersetzungen zu verfassen und seinen Briefen beizulegen bzw. in diese zu integrieren. Er besingt Orte, die er nie gesehen hat und die er allein aus den Briefen Sulzers kennt. Sulzers Briefe sind für seine Schweizer Freunde, die diese kopieren und untereinander zirkulieren lassen, eine der wichtigsten Quellen über den König und seine kriegerischen Aktivitäten, auch weil man den „Gazetten“ nicht immer trauen kann und will. Gerade das Phänomen eines brieflichen Kultes um Friedrich II. und Preußen macht deutlich, wie Briefe als bewegliche Medien Ideen und Konzepte von Wissen, Bildung, Nation, Politik, Sprache und Emotionen tradieren und zugleich deren evozierte Räume tangieren konnten. Briefe wirken kollektiv gedächtnis- und identitätsstiftend über Landesgrenzen hinaus. Die Brutalität preußischer Truppen wird dabei nur selten als problematisch empfunden, sondern oft als heroisch beschrieben. Zugleich machen in Briefen Gerüchte von der Grausamkeit der Gegner die Runde. Dass sich in der russischen Armee „Callmuken“ befinden, die auf dem Schlachtfeld „rohes Menschenfleisch“ gegessen haben sollen, wird sowohl von Gleim als auch von Sulzer in ihren Briefen verbreitet. ${ }^{72}$ Die Herausbildung und Förderung nationaler Stereotypen (z. B. die „grausamesten“73 barbarischen Russen oder die „Hochmüthige Nation"74 Frankreich) wird maßgeblich durch Briefe unterstützt und transferiert.

Kritisch wird es, wenn das Schlachtfeld nicht mehr nur im sicheren Raum des handschriftlichen Briefes existiert, sondern zu einer realen Bedrohung anwächst. So muss Sulzer die Belagerung von Berlin im Jahr 1757 durch russische Truppen hautnah miterleben und der in Briefen und Liedern so oft beschriebene Garten Gleims fällt der Verwüstung durch französische Truppen anheim:

Mein Vergnügen, der Garten, den ich in einem Jahre zu einem kleinen Paradiese gemacht hatte, die Laube, in der mein Alexis [Ramler, Anm. JK] einmahl mit der Nachtigall um die Wette singen solte, der Spatziergang von tausend Linden, der den Garten umschloß pp. alles dis mein liebster Freund ist nicht mehr! Alles, alles ist von dem wütenden Mars in eine Wüsteney verwandelt. ${ }^{75}$

71 Vgl. auch Hüppauf 2003.

72 Sulzer an Bodmer, 20. September 1757, in: Sulzer u. Bodmer 2020, 363; Gleim an Ramler, 6. Januar 1759, in: Gleim und Ramler 1907, 358.

73 Sulzer an Bodmer, 20. September 1757, in: Sulzer u. Bodmer 2020, 362.

74 Sulzer an Bodmer, 11. März 1758, in: Sulzer u. Bodmer 2020, 378.

75 Gleim an Ramler, 7. Oktober 1757, in: Gleim u. Ramler 1907, 293. 
Die lebensweltlich verankerten Räume des empfindsamen Briefes sind Aggression und Gefährdung ausgesetzt. Gleims Halberstädter Garten wird nicht wieder neu angelegt. Sulzers Garten an der Spree, der bis in die erste Hälfte des 19. Jahrhunderts besteht und noch Jahre nach seinem Tod zahlreiche Botaniker anlockt, die sich für sein Arboretum nordamerikanischer Bäume interessieren, fällt ebenso wie Ramlers Haus und Garten städtebaulichen Maßnahmen bzw. dem Zweiten Weltkrieg zum Opfer. Gleims Kirsch- und Walnussbaum oder Ramlers „Levcojen“ und die an sie geknüpften geselligen Zusammenkünfte sind allein in ihren Briefen konserviert.

\section{Schlussbemerkung}

Die Reaktion und Referenzierung auf Räume und Topographien sowie deren Funktionalisierung und semantische Aneignung nehmen innerhalb epistolarer Kommunikation und Praktiken einen hohen Stellenwert ein. Die in Briefen sprachlich und schriftlich konstituierten Räume, die im Falle der hier behandelten Beispiele als konkrete Schreib- und Lektüreorte, Hintergrundszenerie, virtueller Handlungs-, Gesprächs- und Begegnungsraum oder auch als semantische und wirkungsästhetische Bedeutungsträger erscheinen können, überschneiden sich mit epochenspezifischen anthropologischen Entwicklungen und historischen Raumkonzepten und -modellen. Mit Hinblick auf eine Systematisierung und Theoriebildung wäre es sicher für die Briefforschung nicht uninteressant, das, was hier nur exemplarisch und auf einen engen Zeitraum und ausgewählten Quellencorpus begrenzt, ausgeführt werden konnte, in einem größeren und kulturgeschichtlich breiter gefassten Rahmen zu diskutieren.

\section{Literatur}

Benjamin, Walter (1991). „Ich packe meine Bibliothek aus - Eine Rede über das Sammeln (1931)“, in: Gesammelte Schriften. Bd. IV, 1. Hg. v. Tillman Rexroth. Frankfurt a. M.: 388-396.

Breuer, Ingo (2019). „Frauen-Zimmer, Brief-Räume. Über einige Topographien weiblicher Briefkultur im 18. Jahrhundert“, in: „Nur Frauen können Briefe schreiben. “Facetten weiblicher Briefkultur nach 1750. Hg. v. Renata Damp-Jarosz u. Paweł Zarychta. Frankfurt a. M.: 31-47. Bunzel, Wolfgang (2008). „Schreib-/Leseszenen“, in: Der Brief - Ereignis und Objekt. Katalog der Ausstellung im Freien Deutschen Hochstift - Frankfurter Goethe-Museum, 11. September bis 16. November 2008. Hg. v. Anne Bohnenkamp-Renken u. Waltraud Wiethölter. Frankfurt a. M. u. Basel: 237-262. 
Campe, Rüdiger (1991). „Die Schreibszene, Schreiben“, in: Paradoxien, Dissonanzen, Zusammenbrüche. Situationen offener Epistemologie. Hg. v. Hans Ulrich Gumbrecht u. K. Ludwig Pfeiffer. Frankfurt a. M.: 759-772.

Décultot, Elisabeth u. Jana Kittelmann (2020). „Das 18. Jahrhundert im Briefformat. Zur Korrespondenz zwischen Johann Georg Sulzer und Johann Jakob Bodmer“, in: Johann Georg Sulzer - Johann Jakob Bodmer. Briefwechsel. Kritische Ausgabe. Hg. v. Elisabeth Décultot u. Jana Kittelmann unter Mitarbeit v. Baptiste Baumann. 2 Bde. Basel: IX-XLVI.

Der Freundschaftstempel im Gleimhaus zu Halberstadt. Porträts des 18. Jahrhunderts. Bestandskatalog. Bearbeitet v. Horst Scholke. Mit einem Essay v. Wolfgang Adam. Leipzig 2000.

Disselkamp, Martin (2005). „Wein und Liebe, Stahl und Eisen - Anakreontisches und Kriegerisches bei Johann Wilhelm Ludwig Gleim“, in: Anakreontische Aufklärung. Hg. v. Manfred Beetz u. Hans-Joachim Kertscher. Tübingen: 201-221.

Dünne, Jörg u. Stephan Günzel (Hg.) in Zusammenarbeit mit Hermann Doetsch u. Roger Lüdeke (2006). Raumtheorie - Grundlagentexte aus Philosophie und Kulturwissenschaften. Frankfurt a. $M$.

Dünne, Jörg u. Andreas Mahler (Hg.) (2015). Handbuch Literatur und Raum. Berlin u. Boston.

Ehlich, Konrad (1993). „Text und sprachliches Handeln. Die Entstehung von Texten aus dem Bedürfnis nach Überlieferung“, in: Schrift und Gedächtnis. Beiträge zur Archäologie der literarischen Kommunikation. Hg. v. Aleida u. Jan Assmann u. Christof Hardmeier. München: 24-43.

Gleim, Johann Wilhelm Ludwig u. Anna Louisa Karsch (1997). „Mein Bruder in Apoll“. Briefwechsel. Hg. v. Regina Nörtemann u. Ute Pott. 2 Bde. Göttingen.

Gleim, Johann Wilhelm Ludwig u. Karl Wilhelm Ramler (1906-1907). Briefwechsel. Hg. v. Carl Schüddekopf. 2 Bde. Tübingen.

Gleim, Johann Wilhelm Ludwig u. Johann Peter Uz (1898). Briefwechsel. Hg. v. Carl Schüddekopf. Tübingen.

Goethe, Johann Wolfgang von (1997). Sämtliche Werke. Abt. II, 2: Das erste Weimarer Jahrzehnt. Hg. v. Hartmut Reinhardt. Frankfurt a. M.

Hentschel, Uwe (2001). „Besuche in Briefen. Die epistolare Praxis der Anakreontiker und Gellerts Briefreform“, in: Orbis Litterarum 56: 378-395.

Hildebrandt, Annika (2019). Die Mobilisierung der Poesie. Literatur und Krieg um 1750. Berlin u. Boston.

Holm, Christiane (2018). „Tapete“, in: Das 18. Jahrhundert. Lexikon zur Antikenrezeption in Aufklärung und Klassizismus. Hg. v. Joachim Jacob u. Johannes Süßmann. Stuttgart: 945-951.

Hüppauf, Bernd (2003). „Das Schlachtfeld als Raum im Kopf. Mit einem Postscriptum nach dem 11. September 2001“, in: Schlachtfelder - Zur Codierung von Gewalt im medialen Wandel. Hg. v. Steffen Martus, Marina Münkler u. Werner Röcke. Berlin u. Boston: 207-233.

Kittelmann, Jana (2019). „Bist Du allein im Garten, oder ist noch jemand da?“ Wilhelmine Keusenhoff und die Briefkultur der Empfindsamkeit, in: „Nur Frauen können Briefe schreiben“. Facetten weiblicher Briefkultur nach 1750. Hg. v. Ursula Damp-Jarocs u. Paweł Zarychta. Frankfurt a. M.: 61-78.

Kittelmann, Jana (2020a). „Sammeln im Kollektiv. Zur Briefpraxis der Forsters“, in: Weltensammeln. Johann Reinhold und Georg Forster. Hg. v. Elisabeth Décultot, Jana Kittelmann, Andrea Thiele u. Ingo Uhlig. Göttingen: 187-210.

Kittelmann, Jana (2020b). „(Brief-)Lektüren in Gärten“, in: Lektüre und Geschlecht. Zur Situativität des Lesens zwischen Einsamkeit und Geselligkeit. Hg. v. Luisa Banki u. Katrin Wittler. Göttingen: 107-128. 
Kittelmann, Jana (2021). „Gesellige Idylle/Idyllische Geselligkeit als literarisch-kulturelle Praxis um 1750“, in: Paradigmen des Idyllischen. Ökonomie - Ökologie - Artikulation - Gemeinschaft. Hg. v. Nils Jablonski u. Solvejg Nitzke. Bielefeld [im Erscheinen].

Kleist, Ewald Christian von (1881). Briefe von und an Ewald Christian von Kleist. Hg. v. August Sauer. 2 Bde. Berlin.

Klopstock, Friedrich Gottlob (1979). Werke und Briefe. Abt. Briefe I, Bd. 1. Hg. v. Klaus Gronemeyer. Berlin u. New York.

Koebner, Thomas (1977). „Lektüre in freier Landschaft. Zur Theorie des Leseverhaltens im 18. Jahrhundert“, in: Leser und Lesen im 18. Jahrhundert. Hg. v. der Arbeitsstelle Achtzehntes Jahrhundert. Heidelberg: 40-57.

Lacher, Reimar F. (2017). ,Friedrich, unser Held'. Gleim und sein König. Göttingen.

Lee, David (2011). „Amor im Harnisch. Gleim als Anakreontiker und Grenadier“, in: Euphorion 105: 19-50.

Lehnert, Gertrud (2014). „Räume und Affekte. Boudoir und bürgerlicher Innenraum“, in: Räume und Lebensstile im 18. Jahrhundert. Hg. v. Gertrud Lehnert u. Brunhilde Wehinger. Hannover: 13-33.

Neumeyer, Harald (2015). ,, aber alles war rebellisch geworden‘. Das Gasthaus um 1800 Geselligkeit im Ausnahmezustand“, in: Riskante Geselligkeit. Spielarten des Sozialen um 1800. Hg. v. Günther Oesterle u. Torsten Valk. Würzburg: 217-239.

Niedermeier, Michael (2015). „Landschaft/Garten“, in: Handbuch europäische Aufklärung. Hg. v. Heinz Thoma. Heidelberg: 323-334.

Pott, Ute (2013). „Das Tapetenalbum im Gleimhaus Halberstadt“, in: Album. Organisationsform narrativer Kohärenz. Hg. v. Anke Kramer u. Annegret Pelz. Göttingen: 271-277.

Roßbach, Nikola (2015). Wissen, Medium und Geschlecht. Frauenzimmer-Studien zu Lexikographie, Lehrdichtung und Zeitschrift. Frankfurt a. M.

Sauder, Gerhard (1974). Empfindsamkeit. Bd. 1: Voraussetzungen und Elemente. Stuttgart.

Schuster, Jörg (2014). „Kunstleben“. Zur Kulturpoetik des Briefs um 1900 - Korrespondenzen Hugo von Hofmannsthals und Rainer Maria Rilkes. Paderborn.

Sulzer, Johann Georg (1748). Brief an Johann Wilhelm Ludwig Gleim. Museum der deutschen Aufklärung - Gleimhaus Halberstadt: Sign. Hs. A 4072.

Sulzer, Johann Georg (1750). Brief an Wilhelmine Keusenhoff. Freies Deutsches Hochstift Frankfurt a. M.: Sign. Hs. 2999.

Sulzer, Johann Georg (1750-1759). Briefe an Martin Künzli. Studienbibliothek/Winterthurer Bibliotheken: Sign. Ms BRH 512/72.

Sulzer, Johann Georg (1752). Brief an Johann Wilhelm Ludwig Gleim. Museum der deutschen Aufklärung - Gleimhaus Halberstadt: Sign. Hs. A 4126.

Sulzer, Johann Georg u. Johann Jakob Bodmer (2020). Briefwechsel - Kritische Ausgabe. Hg. v. Elisabeth Décultot u. Jana Kittelmann unter Mitarbeit v. Baptiste Baumann. 2 Bde. Basel.

van der Zande, Johan (1999). „Johann Georg Sulzer: Spaziergänge im Berliner Tuskulum“, in: Berliner Aufklärung. Hg. v. Ursula Goldenbaum u. Alexander Košenina. Hannover: 41-68.

Vellusig, Robert (2000). Schriftliche Gespräche. Briefkultur im 18. Jahrhundert. Wien.

Vellusig, Robert (2011). „Aufklärung und Briefkultur. Wie das Herz sprechen lernt, wenn es zu schreiben beginnt“, in: Das achtzehnte Jahrhundert 35,2: 154-171.

Verschragen, Jeroen (2001). Die „stummen Führer“ der Spaziergänger. Über die Wege im Landschaftsgarten. Frankfurt a. M.

Wegmann, Nikolaus (1988). Diskurse der Empfindsamkeit. Zur Geschichte eines Gefühls in der Literatur des 18. Jahrhunderts. Stuttgart. 
